\title{
Demo Abstract: Cross-technology TDMA synchronization using energy pattern beacons
}

\author{
Jan Bauwens, Bart Jooris, Peter Ruckebusch, Domenico Garlisi, Josesph Szurley, Marc Moonen, \\ Spilios Giannoulis, Ingrid Moerman, Eli De Poorter \\ Email: jan.bauwens@intec.ugent.be \\ Ghent University - imec \\ IDLab \\ iGent Tower - Department of Information Technology \\ Technologiepark-Zwijnaarde 15, B-9052 Ghent, Belgium
}

\begin{abstract}
When different technologies use the same frequency bands in close proximity, the resulting interference typically results in performance degradation. Coexistence methods exist, but these are often technology specific and require technology specific interference detection methods. To remove the root cause of the performance degradation, devices should be able to negotiate medium access even when using different technologies. To this end, an architecture that allows cross-technology medium access by means of a Time Division Multiple Access (TDMA) scheme was devised. In order to achieve cross-technology synchronization, which is required for the TDMA solution, an energy pattern beacon is transmitted. The use of energy patterns is sufficiently technology agnostic to allow multiple technologies to negotiate between each other. To showcase the feasibility of crosstechnology synchronization a demo set-up, using IEEE802.15.4 and IEEE802.11 devices in the w-iLab.t testbed, has been created. It demonstrates that the TDMA solution can successfully divide the medium between the different technologies in order to minimize cross-technology interference.
\end{abstract}

\section{INTRODUCTION}

Currently a significant number of wireless technologies use the unlicensed $2.4 \mathrm{GHz}$ Industrial, Scientific and Medical (ISM) frequency bands for wireless communication. The increasing number of devices using the same limited medium has caused a large number of interference issues. Without medium access negotiation, a throughput loss of up to $30 \%$ for IEEE802.11 and 60\% for IEEE802.15.4 is possible when both technologies coexist in the same environment [1]. Within the same technology interference can easily be minimized by designing medium access control (MAC) protocols that intelligently allocate the medium to individual devices. For cross-technology MAC protocols the choices are limited: agreements on medium usage between different technologies is difficult due to a lack of direct communication between devices. Several attempts were made at creating a more advanced architecture that allows cross-technology communication. These architectures were not scalable due to overhead, or were limited to only a couple of technologies [2], [3].

To solve these issue this demo showcases an architecture that combines the use of energy detection and a backbone for cross-technology communication between IEEE802.11 and IEEE802.15.4 devices which results in fair use of the limited wireless medium.

\section{CONTRiBution}

A Time Division Multiple Access (TDMA) solution results in a fairer use of the medium: each node gets its own time share during which only that specific node can access it. To avoid transmission collisions, all nodes should be tightly synchronized to the same reference time which is typically kept by one or more central nodes. TDMA is a proven mechanism that can provide high throughput and is typically very robust. Despite the availability of a TDMA protocol in IEEE802.11 as well as in IEEE802.15.4, a cross-technology TDMA is not easy to implement due to a strong dependence on the distribution of timing information. This timing information can only be transmitted to nodes of the same technology and thus a novel approach needs to be taken to achieve synchronization between wireless technologies.

A radio chip might not be able to interpret a beacon from a different technology, but it can detect when the medium is being accessed by means of energy detection. Even more importantly it can detect when a switch between idle and occupied has occurred on a channel. This mechanism in combination with timing information can be used for synchronization purposes. A central node sends beacons which are simple energy patterns containing a sequence of channel access and idle periods with very specific timings.

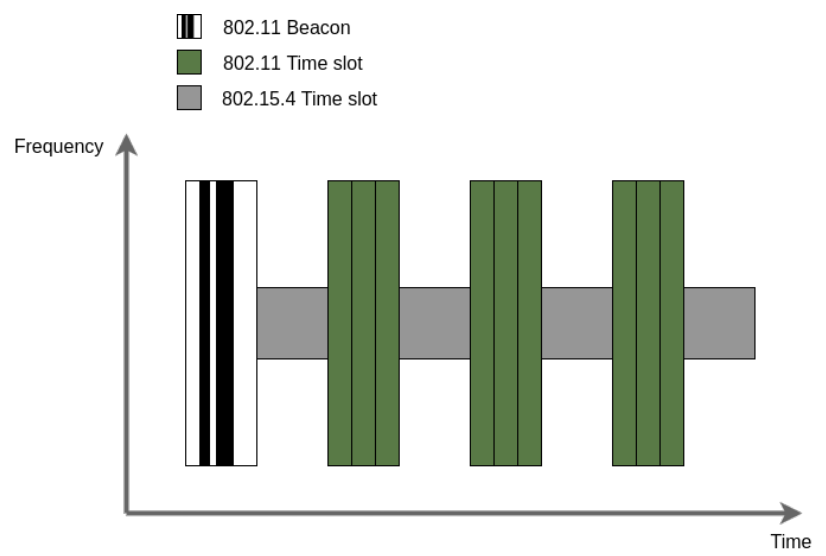

Fig. 1. Cross-technology synchronization and TMDA schedule. Each IEEE802.15.4 slot is followed by three IEEE802.11 slots 
Figure 1 shows an example implementation of such a beacon slot used in the TDMA solution. It is of utmost importance that the pattern should be unique enough to not be labeled as interference, hence the up times shouldnt coincide with commonly used packet lengths [4]. For this implementation, following TDMA slot durations were used: a IEEE802.11 slot corresponds with a slot duration of 2500us, while the IEEE802.15.4 slot duration is 7500us.

To allow other technologies synchronize on the IEEE802.11 beacon, clear channel assessment (CCA) is used. In general the CCA status is used as an indication if a particular channel is available for transmission or not. The CCA check is based on the received signal strength (RSSI) and a configurable threshold. An update is generated every four clock cycles (for IEEE802.15.4 nodes). The MAC protocol is notified of the change of CCA accompanied by the time stamp on which this change has occurred. With some simple subtractions it can be calculated how long the CCA was high/low and if this corresponds with the beacon generated by the access point. For the IEEE802.11 nodes the Wireless MAC Processor (WMP) was used [5]. For the IEEE802.15.4 nodes, a TDMA protocol has been implemented in TAISC, which is an architecture for easy MAC protocol creation [6]. The final TDMA solution is given in figure 1. A beacon slot is followed by the rest of the superframe in which a number of slots is divided among the different nodes in the network independent of which technology the node belongs to. For a more detailed explanation the authors refer to the full paper [8].

\section{DEMO}

During the demo, several aspects of the TDMA solution will be showcased. Real life experiments will be conducted with and without interference present. These tests will be performed on the imec w-iLab.t wireless testbed [7], as it allows for flexible testing of the functionality and performance of wireless networking protocols and systems in a timeeffective way, by providing hardware and the means to install and configure firmware and software on (a selection of) nodes, schedule automated experiments, and collect, visualize and process results. More specifically following hardware will be used:

- IEEE802.11: PC Engines ALIX;

- IEEE802.15.4: RM090 sensor node connected to a Zotac;

- Interference: Zotac with 802.11abgn Wifi card

Several performance parameters will monitored and displayed on live graphs during the duration of the demo. Firstly the synchronization will be evaluated by displaying the number of beacons that were received successfully. The impact of cca threshold and distance to the beacon transmitter will be shown. Secondly the entire TDMA solution will be evaluated via throughput measurements of respectively the IEEE802.11 and IEEE802.15.4 nodes. The spectrum will be monitored in reallife using a USRP device also located in the w-iLab.t testbed (an example result can be seen in figure 2). Several parameters including the interference amount, cca threshold, throughput, etc. will be configurable for the attendees via a user interface.

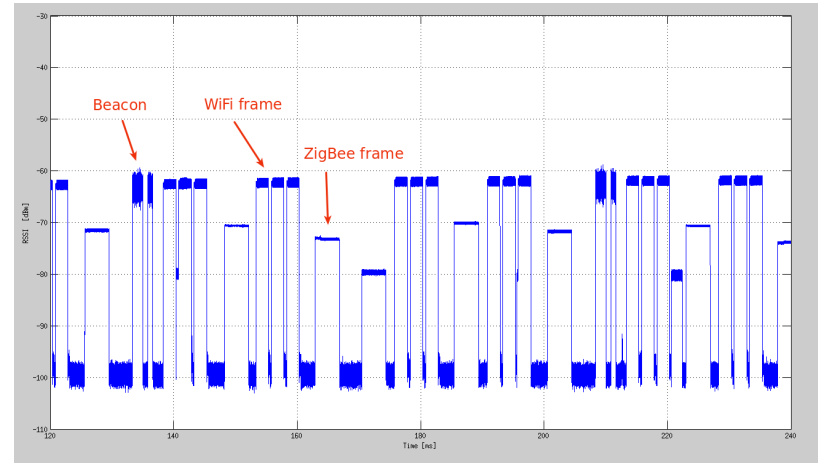

Fig. 2. Cross-technology synchronization and TMDA schedule

The impact will be directly visible via graphs: it will be clear that TDMA using an energy beacon is a good option to divide the medium fairly between devices using different wireless technologies.

\section{CONCLUSION}

This demo showcases the possibility to generate a crosstechnology beacon in a way that other technologies are able to synchronize on it. The impact of parameters as distance to beacon transmitter, cca threshold and interference will be evaluated in an interactive manner. The cross-technology TDMA will be shown as a viable option to mitigate crosstechnology interference.

\section{ACKNOWLEDGMENT}

This work was partially supported by project SAMURAI: Software Architecture and Modules for Unified RAdIo control, and European Commission Horizon 2020 Programme under grant agreement no. 645274 (WiSHFUL).

\section{REFERENCES}

[1] S. Pollin, M. Ergen, M. Timmers, A. Dejonghe, L. Van der Perre, F. Catthoor, I. Moerman, and A. Bahai. "Distributed cognitive coexistence of 802.15. 4 with IEEE802.11.", 2006 1st International Conference on Cognitive Radio Oriented Wireless Networks and Communications. IEEE, 2006.

[2] R. Wang, R. Chang, and H. Chao. "Internetworking between ZigBee/802.15. 4 and IPv6/802.3 network." SIGCOMM Data Communication Festival (2007).

[3] P. Dutta, J. Hui, J. Jeong, S. Kim, C. Sharp, J. Taneja, G. Tolle, K. Whitehouse, and D. Culler. "Trio: enabling sustainable and scalable outdoor wireless sensor network deployments." Proceedings of the 5th international conference on Information processing in sensor networks. ACM, 2006.

[4] K. Chebrolu, and A. Dhekne. "Esense: communication through energy sensing." Proceedings of the 15 th

[5] I. Tinnirello, G. Bianchi, P. Gallo, D. Garlisi, F. Giuliano, and F. Gringoli. "Wireless MAC processors: Programming MAC protocols on commodity hardware." INFOCOM, 2012 Proceedings IEEE. IEEE, 2012.

[6] B. Jooris, J. Bauwens, P. Ruckebusch, P. De Valck, C. Van Praet, I. Moerman, and E. De Poorter. "Taisc: a cross-platform MAC protocol compiler and execution engine." Computer Networks (2016).

[7] S. Bouckaert, W. Vandenberghe, B. Jooris, I. Moerman, and P. Demeester. "The w-iLab. t testbed." International Conference on Testbeds and Research Infrastructures. Springer Berlin Heidelberg, 2010.

[8] J. Bauwens, B. Jooris, P. Ruckebusch, D. Garlisi, J. Szurley, M. Moonen, S. Giannoulis, I. Moerman, E. De Poorter. "Coexistence between IEEE802.15.4 and IEEE802.11 through cross-technology signaling", CNERT, Infocom, 2017 